\title{
genetics
}

\section{Plzf is required in adult male germ cells for stem cell self-renewal}

\author{
F William Buaas ${ }^{1,2,6}$, Andrew L Kirsh ${ }^{1}$, Manju Sharma ${ }^{1}$, Derek J McLean ${ }^{3}$, Jamie L Morris ${ }^{4}$, Michael D Griswold ${ }^{3}$, \\ Dirk G de Rooij ${ }^{5}$ \& Robert E Braun ${ }^{1,2}$
}

\begin{abstract}
Adult germline stem cells are capable of self-renewal, tissue regeneration and production of large numbers of differentiated progeny. We show here that the classical mouse mutant luxoid affects adult germline stem cell self-renewal. Young homozygous luxoid mutant mice produce limited numbers of normal spermatozoa and then progressively lose their germ line after birth. Transplantation studies showed that germ cells from mutant mice did not colonize recipient testes, suggesting that the defect is intrinsic to the stem cells. We determined that the luxoid mutant contains a nonsense mutation in the gene encoding Plzf, a transcriptional repressor that regulates the epigenetic state of undifferentiated cells, and showed that Plzf is coexpressed with Oct4 in undifferentiated spermatogonia. This is the first gene shown to be required in germ cells for stem cell self-renewal in mammals.
\end{abstract}

The luxoid $(l u)$ mutation arose spontaneously in 1950 and was initially characterized for its semidominant limb abnormalities and recessive skeletal and male infertility phenotypes ${ }^{1,2}$. A subsequent report suggested that luxoid mutant males had limited short-term production of normal-looking sperm followed by a rapid loss of all

\footnotetext{
Figure 1 Testis histological phenotype of adult /u/lu mice. (a) Testes from eight-month-old wild-type (right) and /ul/u (left) male mice. (b,c) Hematoxylin and eosin-stained histological cross-sections through the epididymis of an adult /u/lu mouse (b) or wild-type mouse (c). (d) A representative low-power image of a testis from a lu/lu mouse showing numerous tubule phenotypes. Several seminiferous tubules show severe germ cell loss (asterisks). (e) An example of an agametic tubule with only the somatic Sertoli cells (arrowheads) remaining. (f) A mutant tubule containing only elongated spermatids (arrow). Sertoli cells are present along the basal lamina (arrowhead). (g) A mutant tubule with wild-type staging, flanked by tubules with an agametic (asterisk) and an earlier germ-cell generation loss $(\pi)$ phenotype. (h) Low- and (i) high-power images of testis histological sections from 8-month-old controls.
}
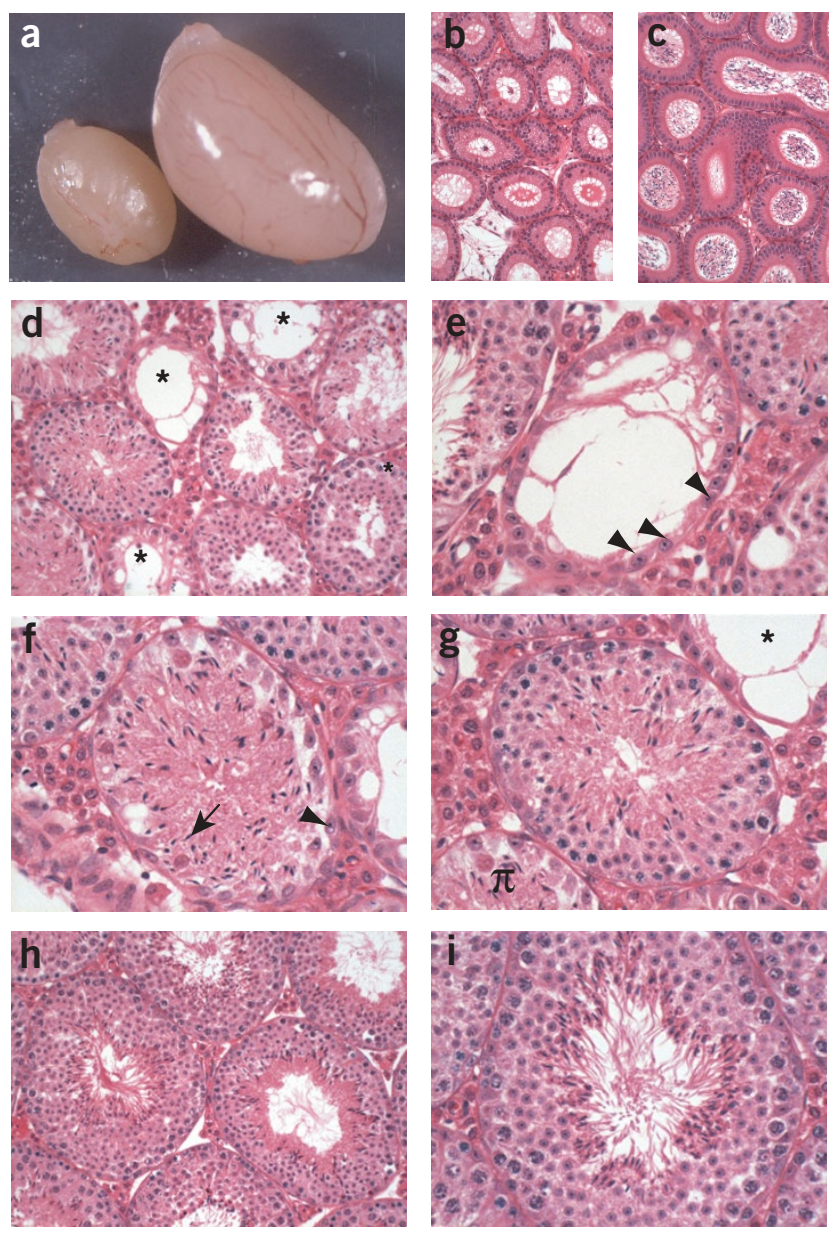

${ }^{1}$ Department of Genome Sciences, Box 357730, 1705 NE Pacific Street, University of Washington School of Medicine, Seattle, Washington 98195, USA. ${ }^{2}$ Molecular and Cellular Biology Program, University of Washington School of Medicine, Seattle, Washington 98195, USA. ${ }^{3}$ Center for Reproductive Biology, School of Molecular Biosciences, Washington State University, Pullman, Washington 99164, USA. ${ }^{4}$ Department of Obstetrics and Gynecology, University of Washington School of Medicine, Seattle, Washington 98195, USA. ${ }^{5}$ Department of Endocrinology, Faculty of Biology, Utrecht University, and Department of Cell Biology, University Medical Center, Utrecht, 3584 CH Utrecht, The Netherlands. ${ }^{6}$ Present address: National Institute for Medical Research, The Ridgeway, Mill Hill, London, England. Correspondence should be addressed to R.E.B. (braun@u.washington.edu). 
germline cells ${ }^{3}$. To reassess the luxoid germ cell phenotype, we established a breeding colony from mice recovered from cryopreservation at The Jackson Laboratory. Testes from 8-month-old homozygous mutants were very small, suggestive of a reduction or elimination of germ cells (Fig. 1a). Histological analysis of epididymides from homozygous mutants showed few sperm relative to controls (Fig. $\mathbf{1 b}, \mathbf{c})$. Examination of sections of testes showed that luxoid mutants had a range of unusual seminiferous tubule phenotypes (Fig. 1d-g) that were not seen in controls (Fig. 1h,i). Many seminiferous tubules were agametic, containing only the somatic Sertoli cells (Fig. 1e). Many tubules lacked one or more germ cell generations. We frequently observed regions of the seminiferous epithelium that contained elongated spermatids but no developmentally younger germ cells (Fig. 1f). Such tubules presumably lost all spermatogonial stem cells and are becoming agametic. We did not observe cohorts of apoptotic germ cells or morphologically abnormal cells being phagocytosed by the Sertoli cells, which could account for the missing germ cells. The luxoid mutation does not disrupt spermatogenesis per se, as tubules with germ cells produce morphologically normal spermatozoa.

We confirmed that germ cells were absent from agametic tubules by staining immunohistochemically for the germ cell nuclear antigen (GCNA), a marker that is expressed in all diploid spermatogonia, meiotic spermatocytes and haploid round spermatids ${ }^{4}$ (Fig. 2a-f). To determine whether the luxoid mutation leads to progressive germ cell loss, we quantified the percentage of GCNA-positive seminiferous tubules in luxoid mutant males and controls at different ages (Fig. 2g).
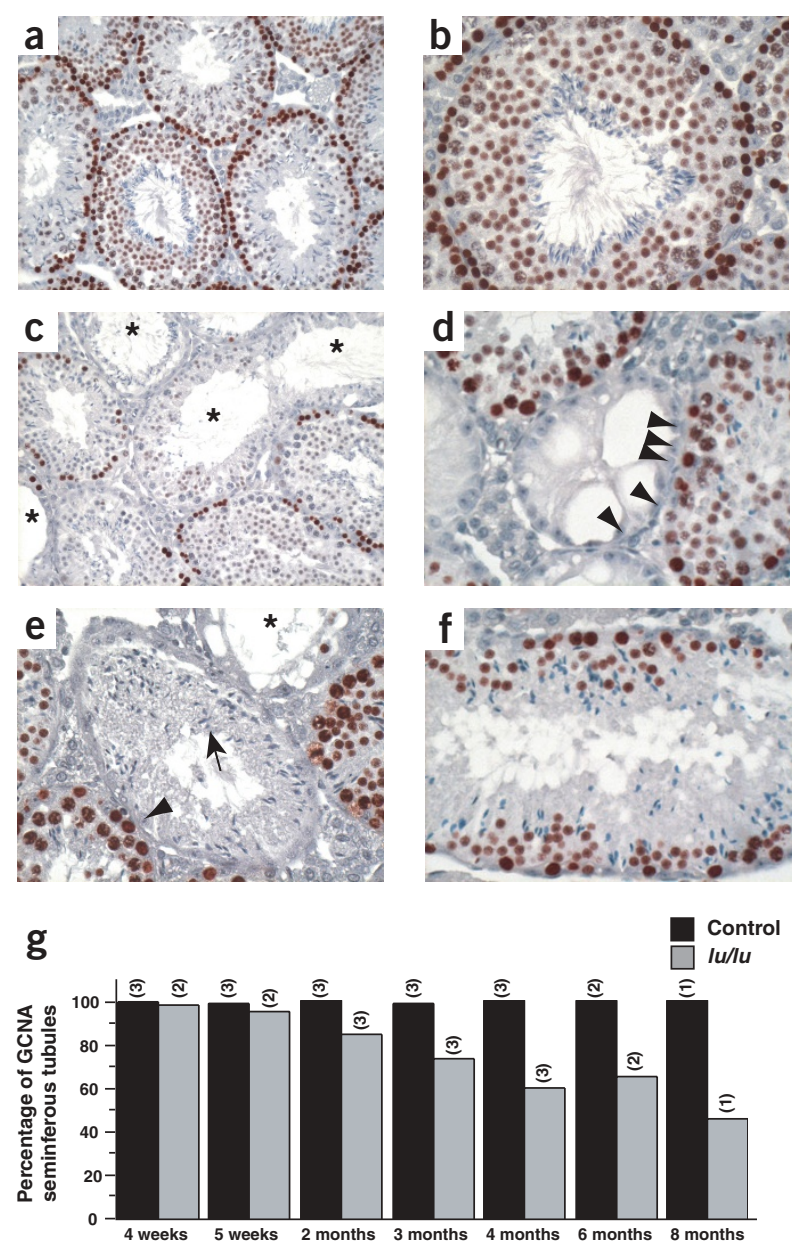

Only tubules that entirely lacked GCNA-positive germ cells were scored as agametic. Four- and five-week-old mutants had slightly fewer GCNA-positive tubules, and older luxoid mutant males had even fewer. Essentially all tubules eventually showed a depletion of GCNA-positive cells.

To directly test if the luxoid mutation affects stem cell self-renewal, and to determine whether the gene defined by the mutation is cellautonomous, we carried out complementary transplantation experiments ${ }^{5}$. In the first set of experiments, we transferred germ cell suspensions prepared from 4 -week-old $l u / l u$ males into recipient $W / W^{N}$ testes, which lack germ cells. We killed recipients 3, 6 and 8 months after transplantation and analyzed their testes histologically by serial sectioning. We examined testes from three recipient mice at each time point but did not detect a single instance of successful colonization (289 histological sections analyzed from nine recipients; Fig. 3a,b). In control experiments, we transferred germ cells from C57BL/6 mice into $W / W^{v}$ testes and obtained successful colonization (data not shown). In the second set of experiments, we determined whether $l u / l u$ males could successfully receive germ cells prepared from Rosa26-lacZ mice. In one of one two-month-old mouse, one of one four-month-old and one of two six-month-old mice, we observed successful colonization of $l u / l u$ testis with $\beta$-galactosidase-positive germ cells (Fig. 3c). Therefore, the somatic compartment of testes in $l u / l u$ mice is capable of supporting spermatogenesis of wild-type germ cells. On the other hand, germ cells from $l u / l u$ males were not able to successfully colonize recipient testes, suggesting that the luxoid mutation directly affects stem cells.

The luxoid mutation arose in a subline of the $\mathrm{C} 3 \mathrm{H} / \mathrm{He}$ inbred strain of mice and was mapped to chromosome 9 (ref. 1). The mutation was then backcrossed onto the C57BL/6 strain before embryos were cryopreserved at generation 35 (N35). To refine the luxoid genetic interval, we carried out an intercross mapping experiment using previously characterized simple sequence-length polymorphic (SSLP) markers ${ }^{6-8}$. This analysis placed the luxoid mutation telomeric to D9Mit256 and centromeric to D9Mit99 (Fig. 4a).

We scanned genomic DNA sequence in the luxoid critical interval for genes that could underlie the luxoid phenotype. Contained in the critical region is $Z f p 145$, which encodes the transcription factor Plzf. The

Figure 2 Loss of the germ cell-specific GCNA marker in mutant testes. (a,c) Low- (200x) and (b,d-f) high- (400x) power photomicrographs of GCNA-stained histological sections. Round spermatids and developmentally younger germ cells are positive for GCNA expression (red), whereas elongated spermatids and all somatic cell types are negative (blue). (a,b) Representative sections from controls showing the nuclear localization and germ cell-specific expression. (c) A representative image of a testis from a Iullu mouse showing numerous tubules with few to no GCNA-positive cells (asterisks). (d) A tubule with no GCNA-positive cells and only the GCNAnegative somatic Sertoli cells (arrowheads). (e) Another mutant tubule lacking any GCNA-positive cells but containing elongated spermatids in a lumenal position (arrow) and somatic Sertoli cells along the basal lamina (arrowhead). A tubule showing an agametic phenotype similar to $\mathbf{d}$ is juxtaposed with this tubule (asterisk). (f) A mutant tubule with several germ cell generations that are GCNA-positive as well as GCNA-negative elongating spermatids. (g) Progressive germ cell loss in testes of Iullu mutants. Testes from each age group were fixed, sectioned and stained for GCNA expression. Testis cross-sections were used to determine the number of GCNA-positive tubules. A seminiferous tubule containing at least a single GCNA-positive cell was classified as GCNA-positive tubule. The number of mice in each age-genotype group is shown in parentheses. More than 70 tubule crosssections were scored for samples taken from all four- and five-week-old mice. All other age groups had a minimum of 240 cross sections scored. The average percentage of GCNA-positive seminiferous tubules are graphed. 
Figure 3 Stem cell transplantation shows that luxoid is cell-autonomous in germline stem cells. (a) Histological section through testis of a W/Wv mouse showing absence of germ cells. (b) Failure to detect successful transplantation of / $u / l u$ germ cells into recipient $W / W^{v}$ testis. Testes from three recipient mice were analyzed at 3, 6 and 8 months of age. A total of 289 histological sections were analyzed from the nine recipients. (c) Intact seminiferous tubules stained with X-gal showing successful transplantation of Rosa26-lacZ germ cells into busulfan-treated lu/lu recipient testis.
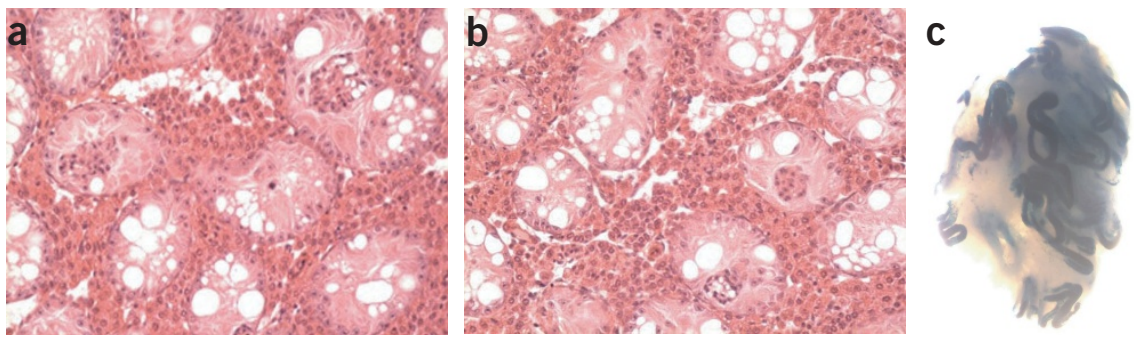

protein contains nine Krüppel-type sequence-specific zinc-finger DNAbinding domains ${ }^{9}$ and a $\mathrm{BTB} / \mathrm{POZ}$ domain that mediates self-association and protein-protein interaction ${ }^{10}$. Its expression pattern in hematopoietic cells, high in multipotential precursors and low in differentiated cells ${ }^{11}$, suggested that Plzf might contribute to stem cell maintenance in the male germ line. In addition, targeted disruption of $Z f p 145$ generates the same spectrum of skeletal phenotypes present in luxoid homozygotes $^{12}$ and causes male sterility ${ }^{13}$. To determine whether $Z f p 145$ underlies luxoid, we amplified by PCR and sequenced its known exons. We found a single nucleotide change in the first coding exon (codon 234) that converts an arginine codon into a nonsense codon (Fig. 4b), resulting in a severely truncated protein containing the $\mathrm{N}$-terminal BTB/POZ domain but lacking all nine zinc-finger domains (Fig. 4c). These data, and the observation that Plzf is expressed in undifferentiated spermatogonia, strongly suggest that mutation of $Z f p 145$ is responsible for the luxoid phenotype. We designated this allele $Z f p 145^{\text {lu }}$.

In the mouse, spermatogonia are classified as A-single (which are thought to be the spermatogonial stem cells), undifferentiated A-paired,
A-aligned, differentiating cells with different cell cycle characteristics $\left(A_{1}, A_{2}, A_{3}, A_{4}\right)$, intermediate and type $B$ spermatogonia ${ }^{14}$. To determine whether Plzf is expressed in spermatogonial stem cells, we carried out immunofluorescence analysis of intact seminiferous tubules isolated from 8-week-old mice. We found that Plzf was expressed in single cells and in pairs and small chains of aligned undifferentiated spermatogonia (Fig. 5a-f). Plzf staining was localized in discrete nuclear foci in cells that had large nuclei and no observable chromocenters. Cells stained positive for Plzf comprised a small fraction of all germline cells stained with DAPI; this was expected, as stem cells are thought to comprise $<0.03 \%$ of all germline cells ${ }^{15}$. As a control, and to confirm that $Z f p 145$ underlies the luxoid phenotype, we showed that Plzf staining was absent in $l u / l u$ males (Fig. 5g,h).

Oct4, a homeobox transcription factor encoded by Pou5f1, is required to maintain the totipotency of embryonic stem cells ${ }^{16,17}$ and is expressed in embryonic primordial germ cells and in spermatogonia in juvenile mice ${ }^{18}$. To determine whether Pou $5 f 1$ is expressed in undifferentiated spermatogonia in adult mice and coexpressed with $Z f p 145$, a

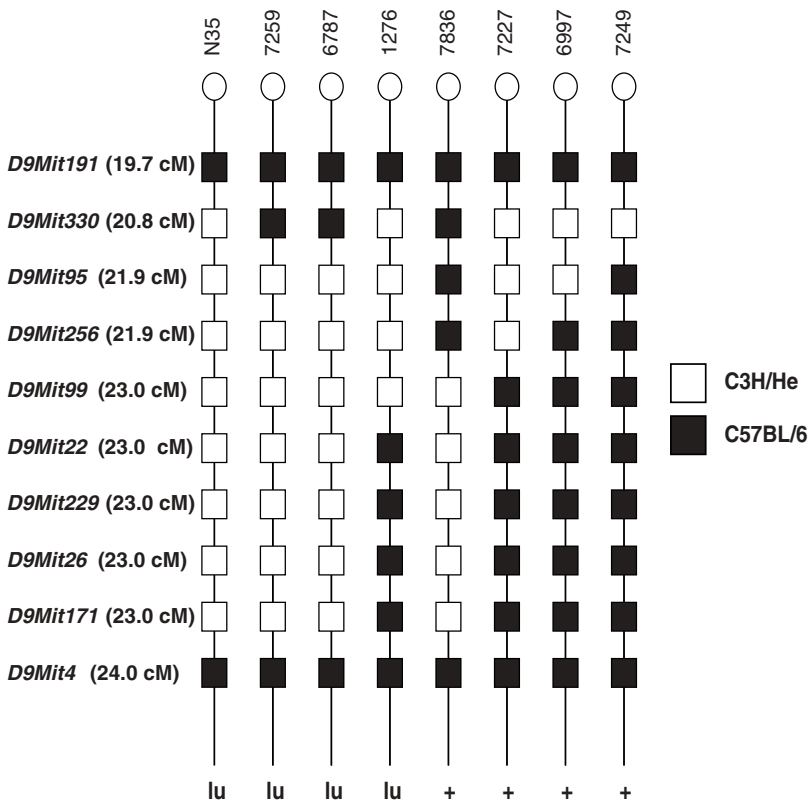

b

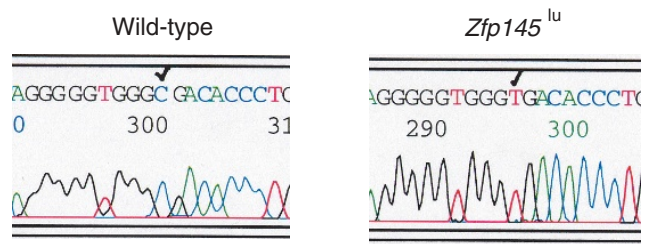

C

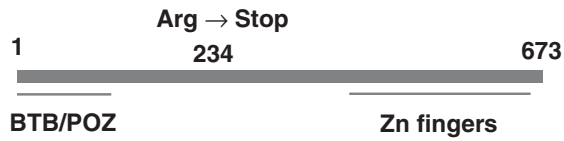

Figure 4 SSLP analysis uncovers residual heterozygosity in C57BL/6.C3H/He-luxoid (N35) mice and defines new recombinant haplotypes. (a) The haplotypes of the N35 and recombinant chromosomes were determined using the SSLP markers shown on the left. The presence (lu) or absence (+) of the luxoid mutation for each haplotype was determined by phenotypic characterization of test-cross progeny and is indicated below the corresponding chromosome. (b) Tracing of DNA sequence of a portion of the first coding exon of Zfp 145 from wild-type and luxoid mice. The position of the single nucleotide change is marked with a check. (c) Schematic showing the location of the BTB/POZ and zinc-finger domains in the wild-type protein and the nonsense mutation at codon 234 in the luxoid mutant. 
we examined expression of green fluorescent protein (GFP) and Plzf in mice carrying a Pou5f1-GFP transgene. This transgene reproduces the endogenous expression pattern of Pou $5 f 1$ in the embryonic and postnatal germ line ${ }^{19}$. Immunofluorescence staining of whole seminiferous tubules using antibodies to GFP and to Plzf showed that the two proteins were coexpressed in spermatogonial stem cells and undifferentiated spermatogonia (Fig. 6a-c). These expression data show that Plzf is expressed in stem cells and confirm our transplantation studies, which indicate that the gene is germ cell-autonomous.

Our initial histological analysis showed that luxoid mutants have progressive germ-cell loss. We used the GFP marker under the control of the Pou $5 f 1$ promoter to verify and extend these findings by carrying out a fluorescence-activated cell sorting (FACS) analysis (Fig. 6d). Even
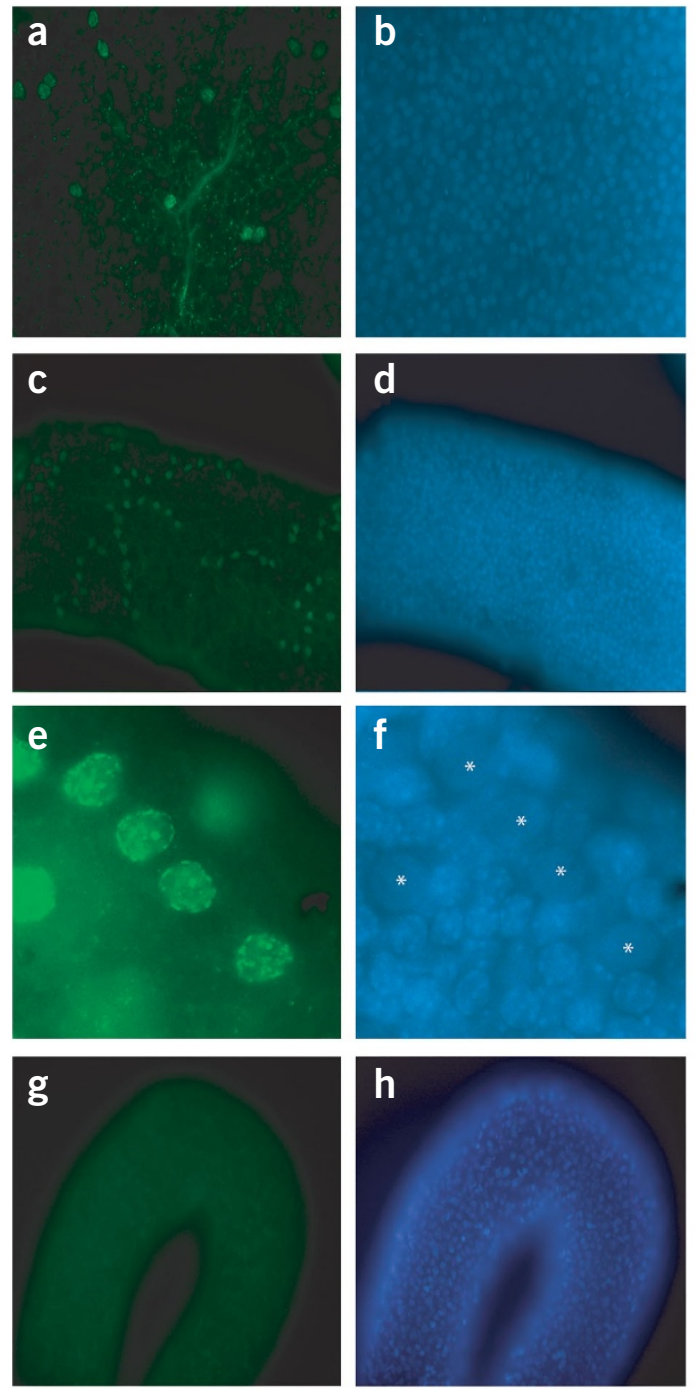

Figure 5 Whole-mount immunofluorescence localization of Plzf in seminiferous tubules of adult mice. (a) View of intact seminiferous tubules stained for Plzf. Single and paired spermatogonia are visible. (b) DAPIstained image of a. (c) Representative example of chains of aligned Plzfpositive spermatogonia. (d) DAPI-stained image of $\mathbf{c}$ showing many nuclei. (e) Enlarged image of Plzf-positive foci. (f) DAPI-stained image of e showing aligned spermatogonia with large nuclei and the absence of chromocenters (asterisks). (g) Absence of Plzf in seminiferous tubules from an 8-week-old luxoid homozygote. (h) DAPI staining of tubule in $\mathbf{g}$ showing that many germline cells are still present. at 8 days of age, $l u / l u$ homozygotes had a significantly lower percentage of GFP-positive sorted cells $(1.0 \pm 0.7)$ than wild types $(7.5 \pm 2.6)$ and heterozygotes $(9.0 \pm 1.04$; values are mean \pm s.e.m., $n=3$ for each genotype; $P<0.01)$. We used semiquantitative RT-PCR to show that there was a slight reduction in the expression of the endogenous Pou $5 f 1$ mRNA in 10-d-old homozygous mice (Fig. 6e) and a more substantial reduction in 8 -week-old mutant males. The reduction in the number of GFP-positive cells in 8-d-old mice suggests that Plzf also functions in the initial allocation of the stem cells in the postnatal testis or, alternatively, that it functions embryonically to affect the number of primordial germ cells.

Previous reports suggested a function for Plzf in cell cycle regulation ${ }^{20}$. One possible mechanism by which mutation of $Z f p 145$ could lead to loss of germline stem cells is by an alteration in the spermato-
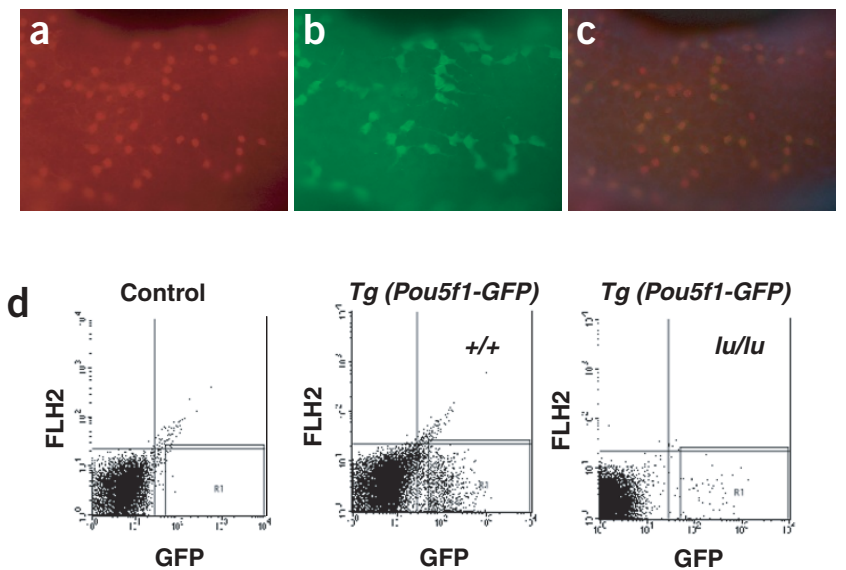

e

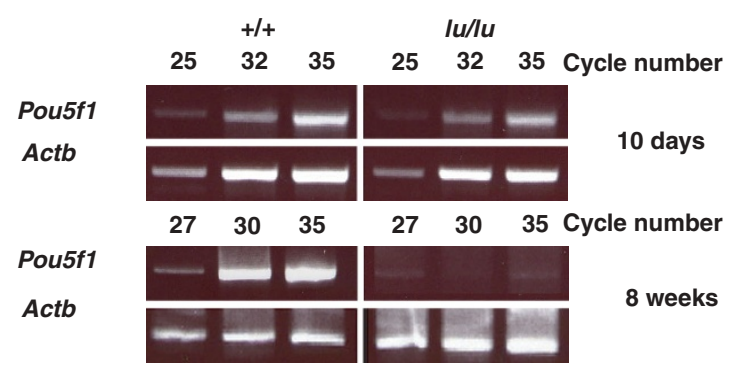

f
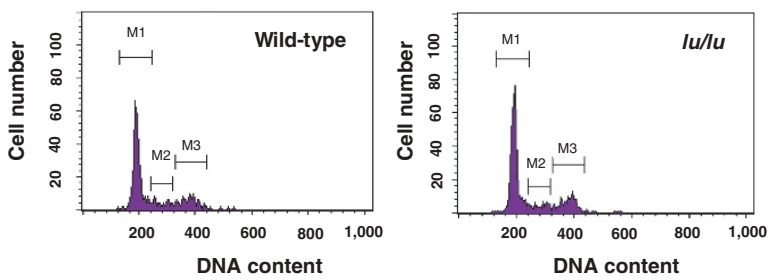

Figure 6 Coimmunofluorescence localization of Plzf and Oct4 in undifferentiated spermatogonia. Detection of antibodies to PIzf (a), GFP (b) and a merge (c) in a 70-d-old transgenic mouse expressing GFP under the control of the Pou5f1 promoter ${ }^{19}$. (d) FACS analysis of the number of GFP-positive cells in 8-d-old wild-type and homozygous luxoid mutant mice. The plot on the left shows the background fluorescence in a nontransgenic mouse. Three mice of each genotype were analyzed. Shown is a representative plot from one mouse of each genotype. Tg, transgenic.

(e) Semiquantitative RT-PCR of the endogenous Pou5f1 and Actb genes. (f) Cell cycle profiles of GFP-positive cells purified from control and luxoid mutant mice by FACS and stained with propidium iodide. Histograms showing the DNA content and cell cycle phases of wild-type and luxoid mutant stem cells. M1, G0/G1; M2, S phase; M3, G2 phase. 
gonial cell cycle. We tested this possibility by measuring the cell cycle profile of GFP-positive cells purified by FACS and found that the cell cycle profiles of $l u / l u$ and wild-type cells were the same at postnatal day 8 (Fig. 6f), suggesting that an altered cell cycle is probably not responsible for the luxoid phenotype.

Plzf was first identified as a chromosomal translocation partner with RAR $\alpha$ in individuals with acute promyelocytic leukemia ${ }^{21}$. It is expressed in immature hematopoietic cells and is downregulated after differentiation ${ }^{11}$. Functional studies in mice suggest that Plzf is required for proper patterning of the axial skeleton and limb buds, and loss of Plzf function leads to anterior-to-posterior homeotic transformations ${ }^{12}$. The BTB/POZ domain of Plzf recruits members of the mammalian Polycomb family (e.g., BMI1), which in turn recruit histone deacetylases to discrete nuclear foci ${ }^{11,22}$. BMI1 is required to maintain adult selfrenewing hematopoietic and leukemic stem cells ${ }^{23,24}$. Similarly, fusion of the BTB/POZ domain of Plzf to RAR $\alpha$ in humans with acute promyelocytic leukemia is thought to maintain myeloid cells in an undifferentiated state by recruiting other nuclear corepressors and histone deacetylases ${ }^{25}$. Thus, Plzf is thought to regulate directly the epigenetic repression of chromatin domains required for cell differentiation ${ }^{22}$.

Our studies support the hypothesis that Plzf maintains the undifferentiated state in cells where it is expressed. Loss of Plzf function shifts the balance between stem cell self-renewal and differentiation, toward differentiation at the cost of self-renewal. The coexpression of the Oct4 transcription factor in undifferentiated spermatogonia, and previous studies showing that it functions in embryonic stem cell renewal ${ }^{16,17}$, suggest that it may also function in adult germline stem cell self-renewal.

\section{METHODS}

Mice. We obtained cryopreserved C57BL/6.C3H/He-luxoid (N35) heterozygotes from The Jackson Laboratory. The luxoid allele arose spontaneously in a $\mathrm{C} 3 \mathrm{H} / \mathrm{He}$ inbred strain, was then backcrossed for 14 generations into the C57BL/10 genetic background and then backcrossed for 21 generations into the C57BL/6J strain before embryos were cryopreserved. We intercrossed male and female C57BL/6.C3H/He-luxoid (N35) heterozygotes to establish a breeding colony and to generate homozygous mutant luxoid males for analysis. Pou5f1GFP mice were previously described ${ }^{19}$.

Histology. We prepared histological sections as previously described ${ }^{26}$.

Immunohistochemistry. We stained paraffin sections with a rat monoclonal antibody against GCNA as previously described ${ }^{26}$.

SSLP analysis. We extracted DNA from tail biopsy samples or obtained it from The Jackson Laboratory. DNA purchased from The Jackson Laboratory was from wild-type $\mathrm{C} 3 \mathrm{H} / \mathrm{HeJ}$ and $\mathrm{C} 57 \mathrm{BL} / 6 . \mathrm{C} 3 \mathrm{H} / \mathrm{He}-\mathrm{luxoid}$ (N35) heterozygous mice. We used these DNAs as templates to amplify SSLPs using sequences from the Massachusetts Institute of Technology genome database. We carried out PCR essentially as described ${ }^{8}$. We radioactively labeled one of the two SSLP primers with $\gamma_{-32}{ }^{32}$-ATP and used it to spike a PCR reaction containing both unlabeled SSLP primers. We separated amplified products on an $8 \%$ urea sequencing gel and then used autoradiography to visualize the products. This analysis defined the luxoid region to between markers D9Mit191 and D9Mit4, a 4.3-cM interval when using the genetic positions based on the Massachusetts Institute of Technology Center for Genome Research SSLP linkage map ${ }^{6-8}$. To refine the luxoid genetic interval, we crossed male and female heterozygotes and scored offspring for the presence of recombinant chromosomes. Two highly informative recombinant chromosomes, 7836 and 7227 , along with chromosome 1276, placed the luxoid mutation telomeric to D9Mit256 and centromeric to D9Mit99.

Whole-mount immunofluorescence. We prepared whole mounts of seminiferous tubules as described ${ }^{27,28}$ with minor modifications. We removed testes from capsules and placed them in a dish of cold phosphate-buffered saline (PBS) in an ice slurry. We dissociated tubule segments from each other and from interstitial material by hand under a dissection microscope, washed them with cold PBS, fixed them overnight at $4{ }^{\circ} \mathrm{C}$ in $4 \%$ paraformaldehyde in PBS, washed them in cold PBS and then dehydrated them through successive 5-min incubations in $25 \%, 50 \%$ and $75 \%$ methanol in PBS and $0.1 \%$ Tween 20 on ice. We washed tubules twice with cold methanol and stored them in methanol at $-20{ }^{\circ} \mathrm{C}$. For antibody staining, we rehydrated the tubules at room temperature in graded methanol, PBS and $0.1 \%$ Tween 20 solutions, washed them with PBS and blocked them in $1 \%$ bovine serum albumin in PBS containing $0.02 \%$ sodium azide for $2 \mathrm{~h}$. We used mouse antibody to Plzf (Oncogene Research) at 1:50 dilution in $0.5 \%$ bovine serum albumin in PBS with $0.02 \%$ sodium azide overnight at room temperature and then washed the tubules several times with PBS at room temperature. We then incubated the tubules with an Alexa Fluor 488-conjugated goat antibody to mouse (Molecular Probes) at 1:1,000 dilution for $2 \mathrm{~h}$ at room temperature. After washing them with PBS, we stained the tubules for 10 min with DAPI in PBS, washed them briefly in PBS and mounted them in Vectashield mounting medium (Vector Laboratories).

Donor mice and cell preparation. Protocols for the use of mice in these experiments were approved by the Washington State University Animal Care and Use Committee and in accord with National Institutes of Health standards established by the Guidelines for the Care and Use of Experimental Animals. We recovered donor cells from the transgenic mouse line C57BL/6.129$\operatorname{TgR}$ (Rosa26)26Sor originally purchased from the Jackson Laboratory and maintained at Washington State University, and from luxoid mutant mice maintained at the University of Washington. Rosa26-lacZ mice express the Escherichia coli $\beta$-galactosidase protein in all stages of spermatogenesis, and cells stain blue when incubated with 5 -bromo-4-chloro-3-indoyl- $\beta$-D-galactoside (X-gal). Therefore, germ cells from Rosa26-lacZ mice that colonize recipient testes can be visualized by a blue color after staining with X-gal. Mice were housed in a standard animal facility with free access to food and water.

We enzymatically digested donor testes from Rosa26-lacZ or luxoid mice as described ${ }^{29}$ with modifications. We removed testes, immersed them in Hank's buffer, removed the tunica and transferred the testes to tubes containing digestion medium ( $0.5 \mathrm{mg} \mathrm{m}^{-1}$ of collagenase type IV (Sigma), $0.25 \mathrm{mg} \mathrm{ml}^{-1}$ of trypsin (GibcoBRL), $0.05 \mathrm{mg} \mathrm{ml}^{-1}$ of DNase (Sigma) in Ca- and Mg-free Hank's buffer ( $\mathrm{pH}$ 7.4)). We digested testes by shaking for $15 \mathrm{~min}$ at $33^{\circ} \mathrm{C}$ to dissociate tubules. We transferred the tube containing the tubule suspension to ice and allowed the tubules to sediment for $5 \mathrm{~min}$. We removed the supernatant, added fresh digestion medium and digested the tubules by shaking for $15 \mathrm{~min}$ at $37^{\circ} \mathrm{C}$. After the second digestion, we gently pipetted the cell suspension for several minutes and centrifuged it at $500 \mathrm{~g}$ for $4 \mathrm{~min}$. We resuspended cells in Hank's buffer containing $0.3 \mathrm{mg} \mathrm{ml}^{-1}$ soybean trypsin inhibitor (Sigma) and determined cell concentrations with a hemocytometer. We centrifuged the cell suspension at $500 \mathrm{~g}$ for $4 \mathrm{~min}$ and resuspended it in EKRB medium ${ }^{29}$ containing $0.03 \%$ trypan blue (GibcoBRL) at a concentration of $10^{7}$ cells per ml.

Recipient mice and analysis. We transplanted donor cells into testes of luxoid mice 4 weeks after treatment with busulfan ( $40 \mathrm{mg}$ per $\mathrm{kg}$ body weight) or testes of $W / W^{\mathrm{v}}$ mice (Jackson Laboratory, WBB6F1/J-Kit ${ }^{\mathrm{W}} / \mathrm{Kit}^{\mathrm{Wv}}$ ). We injected germ cells from Rosa26-lac $Z$ mice into luxoid mice. $W / W^{v}$ mice have no endogenous spermatogenesis due to a mutation in the $c$-Kit gene, and so we used them as recipients for luxoid germ cells. We made a midline incision and exposed the testis. We made a small hole in the connective tissue enclosing the efferent tubules and inserted a glass needle containing $4 \mu \mathrm{l}$ (70,000 cells) of the germ cell suspension into the rete testis through the efferent tubules. We injected the cells into the seminiferous tubules through the rete testis, replaced the testis and allowed the mouse to recover. We tried to inject all $4 \mu$ l of the cell suspension into each recipient testis, but when this did not occur, we determined the volume injected and accounted for this when we evaluated the recipient testis for colonization.

We killed recipient mice by cervical dislocation and removed testes, fixed them in $4 \%$ paraformaldehyde in PBS ( $\mathrm{pH}$. 7.4) for $1 \mathrm{~h}$ on ice and washed them twice in $\beta$-galactosidase buffer $(0.2 \mathrm{M}$ sodium phosphate $(\mathrm{pH} 7.3)$, $2 \mathrm{mM} \mathrm{MgCl}, 0.02 \%$ Nonidet P-40 and $0.01 \%$ sodium deoxycholate) for $30 \mathrm{~min}$. We stained recipient luxoid mice injected with Rosa26-lacZ germ cells for $\beta$-galactosidase-positive cells by incubating testes in $\beta$-galactosidase stain solution ( $\beta$-galactosidase buffer containing $20 \mathrm{mM}$ potassium ferricyanide, 
$20 \mathrm{mM}$ potassium ferrocyanide and $1 \mathrm{mg} \mathrm{ml}^{-1}$ of X-gal). We used histological analysis to evaluate germ cells in donor testes and identify the location and type of germ cells expressing $\beta$-galactosidase in recipient testes. We handled testis samples not stained with X-gal using standard techniques and counterstained them with hematoxylin. Recipient testis samples stained with X-gal were passed through a series of ethanol solutions for complete dehydration and washed twice for $2 \mathrm{~h}$ with Histo-Clear (National Diagnostics) at room temperature. We transferred samples to liquid paraffin $\left(55^{\circ} \mathrm{C}\right)$ and incubated them overnight. We changed the liquid paraffin and incubated the samples at $55^{\circ} \mathrm{C}$ for an additional $2 \mathrm{~h}$. We placed the samples in blocks, cut $6-\mu \mathrm{m} \mathrm{sec}-$ tions and placed them on slides. We removed the paraffin with Histo-Clear (National Diagnostics) to avoid fading of blue X-gal stain with xylenes.

FACS. We carried out cell sorting and cell cycle analysis using FACStar cell sorter equipped with CELL Quest software (Becton Dickinson). We sorted GFP-positive cells from total testicular cells prepared from juvenile mice, resuspended them in PBS containing $2 \%$ bovine serum albumin. We fixed the sorted cells in $70 \%$ ethanol, incubated them with propidium iodide and RNase A at $37^{\circ} \mathrm{C}$ for 30 min and analyzed the DNA content and cell cycle phase by FACScan.

\section{ACKNOWLEDGMENTS}

We thank K. McLaughlin for supplying the Pou5f1-GFP mice and A. Dearth,

I. Mohar and I. Leaf for technical assistance. This work was supported by a grant to

R.E.B. from the National Institute of Child Health and Human Development's

Contraceptive Development Research Centers Program.

\section{COMPETING INTERESTS STATEMENT}

The authors declare that they have no competing financial interests.

Received 9 February; accepted 9 April 2004

Published online at http://www.nature.com/naturegenetics/

1. Green, M.C. Luxoid, a new hereditary leg and foot abnormality in the house mouse. J. Hered. 46, 91-99 (1955).

2. Forsthoefel, P.F. The skeletal effects of the luxoid gene in the mouse, including its interactions with the luxate gene. J. Morphol. 102, 247-287 (1958).

3. Johnson, D.R. \& Hunt, D.M. Hop-sterile, a mutant gene affecting sperm tail development in the mouse. J. Embryol. Exp. Morphol. 25, 223-236 (1971).

4. Enders, G.C. \& May, J.J. Developmentally regulated expression of a mouse germ cell nuclear antigen examined from embryonic day 11 to adult in male and female mice. Dev. Biol. 163, 331-340 (1994).

5. Brinster, R.L. \& Avarbock, M.R. Germline transmission of donor haplotype following spermatogonial transplantation. Proc. Natl. Acad. Sci. USA 91, 11303-11307 (1994).

6. Copeland, N.G. et al. Genome maps IV 1993. Wall chart. Science 262, 67-82 (1993).
7. Dietrich, W.F. et al. A genetic map of the mouse with 4,006 simple sequence length polymorphisms. Nat. Genet. 7, 220-245 (1994).

8. Dietrich, W.F. et al. A comprehensive genetic map of the mouse genome. Nature $\mathbf{3 8 0}$ 149-152 (1996).

9. Li, J.Y. et al. Sequence-specific DNA binding and transcriptional regulation by the promyelocytic leukemia zinc finger protein. J. Biol. Chem. 272, 22447-22455 (1997).

10. Bardwell, V.J. \& Treisman, R. The POZ domain: a conserved protein-protein interaction motif. Genes Dev. 8, 1664-1677 (1994).

11. Reid, A. et al. Leukemia translocation gene, PLZF, is expressed with a speckled nuclear pattern in early hematopoietic progenitors. Blood 86, 4544-4552 (1995).

12. Barna, M., Hawe, N., Niswander, L. \& Pandolfi, P.P. PLZF regulates limb and axia skeletal patterning. Nat. Genet. 25, 166-172 (2000).

13. Hawe, N., Soares, V., Niswander, L., Cattoretti, G. \& Pandolfi, P.P. Targeted disruption of the Plzf gene of acute promyelocytic leukemia results in phocomelia and altered spermatatogenesis. Blood 88, 1152 (1996).

14. de Rooij, D.G. Proliferation and differentiation of spermatogonial stem cells. Reproduction 121, 347-354 (2001).

15. Tegelenbosch, R.A. \& de Rooij, D.G. A quantitative study of spermatogonial multiplication and stem cell renewal in the $\mathrm{C} 3 \mathrm{H} / 101 \mathrm{~F} 1$ hybrid mouse. Mutat. Res. 290, 193-200 (1993).

16. Nichols, J. et al. Formation of pluripotent stem cells in the mammalian embryo depends on the POU transcription factor OCT4. Cel/ 95, 379-91 (1998).

17. Niwa, H., Miyazaki, J. \& Smith, A.G. Quantitative expression of OCT3/4 defines differentiation, dedifferentiation or self-renewal of ES cells. Nat. Genet. 24, 372-376 (2000).

18. Pesce, M., Gross, M.K. \& Scholer, H.R. In line with our ancestors: OCT4 and the mammalian germ. Bioessays 20, 722-32 (1998).

19. Szabo, P.E., Hubner, K., Scholer, H. \& Mann, J.R. Allele-specific expression of imprinted genes in mouse migratory primordial germ cells. Mech. Dev. 115, 157-60 (2002).

20. Shaknovich, R. et al. The promyelocytic leukemia zinc finger protein affects myeloid cell growth, differentiation, and apoptosis. Mol. Cell. Biol. 18, 5533-5545 (1998).

21. Chen, Z et al. Fusion between a novel Kruppel-like zinc finger gene and the retinoic acid receptor-alpha locus due to a variant $\mathrm{t}(11 ; 17)$ translocation associated with acute promyelocytic leukaemia. EMBO J. 12, 1161-1167 (1993).

22. Barna, M. et al. PLZF mediates transcriptional repression of HoxD gene expression through chromatin remodeling. Dev. Cel/ 3, 499-510 (2002).

23. Lessard, J. \& Sauvageau, G. Bmi-1 determines the proliferative capacity of normal and leukaemic stem cells. Nature 423, 255-260 (2003).

24. Park, I.K. et al. Bmi-1 is required for maintenance of adult self-renewing haematopoietic stem cells. Nature 423, 302-305 (2003).

25. Lin, R.J. et al. Role of the histone deacetylase complex in acute promyelocytic leukaemia. Nature 391, 811-814 (1998).

26. Nadler, J.J. \& Braun, R.E. Fanconi anemia complementation group C is required for proliferation of murine primordial germ cells. Genesis 27, 117-123 (2000).

27. Rosen, B. \& Beddington, R.S. Whole-mount in situ hybridization in the mouse embryo: gene expression in three dimensions. Trends Genet. 9, 162-167 (1993).

28. Ohmura, M. et al. Increment of murine spermatogonial cell number by gonadotropinreleasing hormone analogue is independent of stem cell factor C-KIT signal. Biol. Reprod. 68, 2304-2313 (2003).

29. O'Brien, D.A. \& Bellvé, A.R. Protein constituents of the mouse spermatozoon. Dev. Biol. 75, 405-418 (1980). 